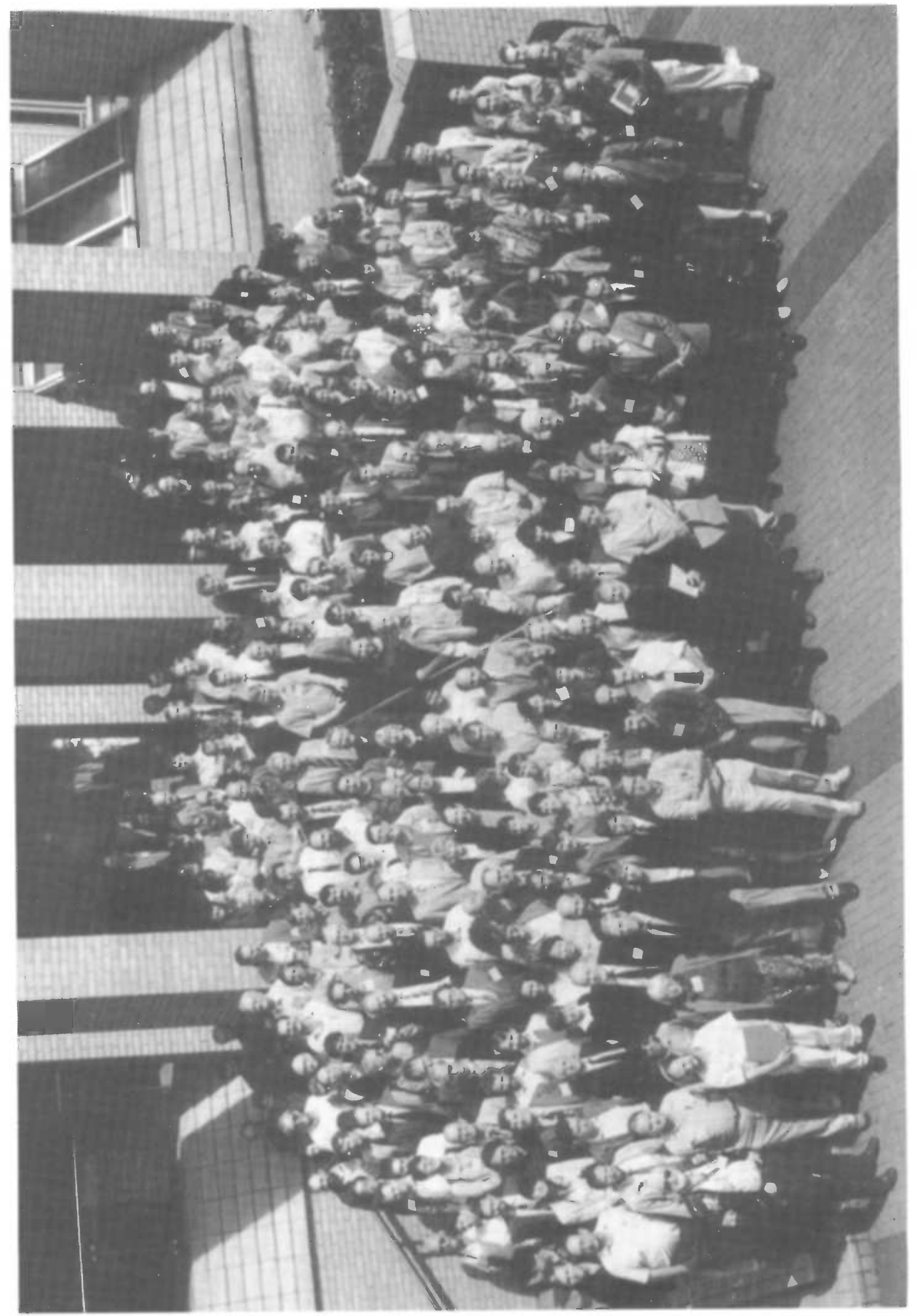




\section{CONFERENCE PARTICIPANTS}

Gerald Aardsma

412 North Mulberry

Loda, Illinois 60948 USA

Tel: 217-386-2247

Mohan Abeyratne

Quaternary Dating Research Centre

Research School of Pacific Studies

Australian National University

Canberra, ACT 0200 Australia

Tel: 61-6-249-3685; Fax: 61-6-249-0738

Cees Alderliesten

R.J. van de Graaff Laboratorium

Rijksuniversiteit Utrecht

P.O. Box 80.000

Princetonplein 5

NL-3508 TA Utrecht, The Netherlands

Tel: 31-30-53 2238; Fax: 31-30-51 8689

Marisa Alessio

Dipartimento di Fisica

Università "La Sapienza"

Piazzale Aldo Moro, 2

I-00185 Rome, Italy

Fax: 39-6-4957697

Janet Ambers

Department of Scientific Research

British Museum

Great Russell Street

London, WC1B 3DG, England

Tel: 44-171 323 8332; Fax: 44-171 3238276

Ramon Aravena

Department of Earth Sciences

University of Waterloo

Waterloo, Ontario, N2L 3G1 Canada

Tel: 519-885-1211 x 5652; Fax: 519-746-7484

E-mail: roaraven@sciborg.uwaterloo.ca

Maurice Arnold

Centre des Faibles Radioactivités

Avenue de la Terrasse

F-91198 Gif sur Yvette CEDEX, France

Tel: 331698235 25; Fax: 33169823568

E-mail: arnold@cfr.cnrs-gif.fr

Kh. A. Arslanov

Institute of Geography

St. Petersburg State University

Srednyi Prospect 41

St. Petersburg 199004 Russia

Tel: 7-812-218-9023 x 73; Fax: 7-812-218-1346
P. J. Ashmore

Historic Scotland

20 Brandon Street

Edinburgh, EH3 5RA, Scotland

Tel: 44-131 244; Fax: 44-131 244

Mike Baillie

Palaeoecology Centre

School of Geosciences

The Queen's University of Belfast

Belfast, BT7 1NN, Northern Ireland

Tel: 44-1232-335 147

E-mail: pag0032@v2.qub.ac.uk or

m.baillie@queens-belfast.ac.uk

John Barber

AOC (Scotland) Limited

4 Lochend Road

Edinburgh, EH6 8BR, Scotland

Mike Barbetti

NWG MacIntosh Centre for

Quaternary Dating

Madsen Building F09

University of Sydney

Sydney, NSW 2006 Australia

Tel: 61-2-692 3993; Fax: 61-2-552 1967

E-mail:mike@emu.su.oz.au

Edouard Bard

CEREGE

Université d'Aix-Marseille III

FU CNRS 17

Europôle de l'Arbois, BP 80

F-13545 Aix en Provence CEDEX 4, France

Tel: 33429715 61; Fax: 3342971505 or 1540

E-mail: ebard@arbois.cerege.fr

Paolo Bartolomei

ENEA, INN-FIS-DIAF

Via dei Colli 16

I-40136 Bologna, Italy

Tel: 39-51-498111; Fax: 39-51-498151

Alex Bayliss

Ancient Monuments Laboratory

English Heritage

Fortress House

23 Savile Row

London, W1X 1AB, England

Tel: 44-171 973 3000; Fax: 44-171 9733001

E-mail: alex@eng-h.gov.uk 
Valentina Bazarova

Pacific Institute of Geography

Radio Street 7

Vladivostok 690041 Russia

Peter Becker-Heidmann

Institut für Bodenkunde

Universität Hamburg

Allende-Platz 2

D-20146 Hamburg, Germany

Tel: 49-40-4123 2003; Fax: 49-40-4123 2024

pbeckerh@rrz.uni-hamburg.de

Jürg Beer

Swiss Federal Institute for Environmental

Science and Technology (EAWAG)

Überlandstrasse 133

CH-8600 Dübendorf, Switzerland

Tel: 411823 5111; Fax: 4118235210

E-mail: beer@eawag.ch

Roelf Beukens

IsoTrace Laboratory

University of Toronto

60 St. George Street

Toronto, Ontario, M5S 1AZ, Canada

Tel: 416-978-4628; Fax: 416-978-4711

isotrace@physics.utoronto.ca

Elisabetta Boaretto

Ralah Institute of Physics

Hebrew University

91904 Givat Ram

Jerusalem, Israel

Roland Bol

Institute for Grassland and

Environmental Research

North Wyke, Okehampton

Devon, EX20 2SB, England

Georges Bonani

Institute für Teilchenphysics

ETH-Hönggerberg

CH-8093 Zürich, Switzerland

Tel: 411633 2043; Fax: 4116331067

E-mail: bonani@imp.phys.ethz.ch

Sheridan Bowman

Department of Scientific Research

British Museum

Great Russell Street

London, WC1B 3DG, England

Tel: 44-171-323 8669; Fax: 441-171-323 8276

E-mail: ezbmxxx@uk.ac.ulcc.vmsfe
Carl A.M. Brenninkmeijer

Max Planck Institute of Chemistry

P.O. Box 3060

D-55020 Mainz, Germany

Tel: 49-6131-305 458; Fax: 49-6131-305 511

Wallace S. Broecker

Lamont-Doherty Earth Observatory

Columbia University

Palisades, New York 10964 USA

Tel: 914-365-8413; Fax: 914-365-8155

E-mail:broecker@lamont.ldeo.columbia.edu

Tom Brown

Center for Accelerator Mass Spectrometry (CAMS)

Lawrence Livermore National Laboratory

P.O. Box 808, L-397

Livermore, California 94551 USA

Tel: 510-423-8507; Fax: 510-423-7884

E-mail: tabrown@llnl.gov

Hendrik J. Bruins

The Jacob Blaustein Institute for

Desert Research

Ben-Gurion University of the Negev

Sede Boker Campus 84993 Israel

Tel: 972-7-565736; Fax: 972-7-555058

E-mail: soc-stud@bgumail.bgu.ac.il

George S. Burr

NSF-Arizona AMS Facility

Department of Physics

The University of Arizona

Tucson, Arizona 85721 USA

Tel: 520-621-8411; Fax: 520-621-9619

E-mail: burr@ccit.arizona.edu

Michael Buzinny

Ukrainian Research Center for

Radiation Medicine

Laboratory of Radioecology

Melnikova 53

254050 Kiev, Ukraine

Tel: 744-2139629; Fax: 744-2137192

E-mail: buz@rpi.kiev.ua

William F. Cain

Chemistry Department

Loyola Marymount University

7101 W. 80th Street

Los Angeles, California 90045-2699 USA

Tel: 310-338-7500; Fax: 310-338-2905

E-mail: wcain@lmumail.lmu.edu 
Israel Carmi

Department of Environmental Sciences and Energy Research

Weizmann Institute of Science

76100 Rehovot, Israel

Tel: 9728 342544; Fax: 9728344124

cicarmii@weizmann.weizmann.ac.il

Maobai Chen

Shanghai Institute of Nuclear Research

Academica Sinica

P.O. Box 800204

Shanghai 201800 China

Tel: 9530 998213; Fax: 86219528021

Christopher Collins

Department of Biology

Silwood Park

Ascot

Berkshire, SL5 7PY, England

E-mail: c.collins@ic.ac.uk

\section{Gordon T. Cook}

Radiocarbon Dating Laboratory

Scottish Universities Research \& Reactor Centre (SURRC)

Scottish Enterprise Technology Park

East Kilbride, G75 0QF, Scotland

Tel: 44-13552-23332; Fax: 44-13552-29898

Mick Cooper

Department of Environmental Sciences

University of Plymouth

Drake Circus

Plymouth, PL4 8AA, England

Tel: 44-1752 233034; Fax: 44-1752 233035

Richard Cresswell

Department of Nuclear Physics

Research School of Physical Sciences

Australian National University

Canberra, ACT 0200 Australia

Tel: 616249 5179; Fax: 6162490748

E-mail: rgc103@nuc.anu.edu.au

Lloyd A. Currie

National Institute of Standards

and Technology

Gaithersburg, Maryland 20899 USA

Tel: 301-975-3919; Fax: 301-975-3845
Kaimei Dai

Department of Physics

Nanjing University

22 Hankou Road

Nanjing 210008 China

Tel: $86-37551$

Magnar Dalland

4 Lochend Road

Edinburgh, EH6 8BR, Scotland

Tel: 44-131-555 4425; Fax: 44-131-551 4426

Paul E. Damon

Department of Geosciences

The University of Arizona

Tucson, Arizona 85721 USA

Tel: 520-621-4659; Fax: 520-621-2672

E-mail: pedamon@geo.arizona.edu

Shelley Danzer

Laboratory of Tree-Ring Research

The University of Arizona

Tucson, Arizona 85721 USA

Tel: 520-621-3846; Fax: 520-621-8229

E-mail: sdanzer@ccit.arizona.edu

A.F.M. de Jong

R.J. van de Graaff Laboratorium

Rijksuniversiteit Utrecht

Princetonplein 5

P.O. Box 80.000

NL-3508 TA Utrecht, The Netherlands

Tel: 313053 2238; Fax: 3130518689

Emmanuelle Delqué Kolic

Centre de Datation par le RadioCarbone

Université Claude Bernard Lyon 1

43 Boulevard du 11 Novembre 1918

Bâtiment 217

F-69622 Villeurbanne CEDEX, France

Tel: 33724482 57; Fax: 3372431317

Valentin Dergachev

A.F. Ioffe Physico-Technical Institut

Russian Academy of Sciences

Polytechnichskaya 26

St. Petersburg 194021 Russia

E-mail: ermolaev@mz.ioffe.rssi.ru 
Michelle Dodds

Radiocarbon Dating Laboratory

Palaeocology Centre

School of Geosciences

The Queen's University of Belfast

Belfast, BT7 1NN, Northern Ireland

Tel: 44-1232-335141; Fax: 44-1232-315779

Douglas J. Donahue

NSF-Arizona AMS Facility

Department of Physics

The University of Arizona

Tucson, Arizona 85721 USA

Tel: 520-621-2480; Fax: 520-621-9619

E-mail:djd@physics.arizona.edu

Quentin Dresser

Department of Geography

University of Wales, Swansea

Singleton Park, Swansea

West Glamorgan, SA2 8PP, Wales

Tel: 44-1792-295148; Fax: 44-1792-205556

E-mail: p.q.dresser@swansea.ac.uk

Ellen Druffel

Radiocarbon Laboratory

Department of Earth Systems Science

University of California, Irvine

PSRF 207

Irvine, California 92717 USA

Tel: 714-824-8794; Fax: 714-824-3256

E-mail: edruffel@uci.edu

Andrew Dugmore

Department of Geography

University of Edinburgh

Drummond Street

Edinburgh, EH8 9XP, Scotland

Tel: 44-131-650 2565; Fax: 44-131-650 2524

E-mail: office@geovax.ed.ac.uk

Warren Eastwood

Institute of Earth Studies

University of Wales

Aberystwyth

Dyfed, SY23 3DB, Wales

Tel: 44-1970 622653

E-mail: wwen@aber.ac.uk
Timothy Eglinton

Fye Laboratory

Marine Chemistry \& Geochemistry Department

Woods Hole Oceanographic Institution

Woods Hole, Massachusetts 02543 USA

Tel: 508-289-2627; Fax: 508-457-2164

E-mail: teglinton@whoi.edu

Sigurður Einarsson

Multi-Detector Systems Ltd.

Hverfisgata 8-10

IS-101 Reykjavík, Iceland

Tel: 354-1-11244; Fax: 354-1-11273

Roos Eisma

c/o Klaas van der Borg

van de Graaff Laboratorium

Rijksuniversiteit Utrecht

Princetonplein 5

P.O. Box 80.000

NL-3508 TA Utrecht, The Netherlands

Tel: 31206364588

E-mail: rosine@lagavulin.xs4all.nl

Kimberley Tanner Elliott

RADIOCARBON

Department of Geosciences

The University of Arizona

4717 E. Ft. Lowell Road

Tucson, Arizona 85712 USA

Tel: 520-881-2722; Fax: 520-881-0554

E-mail:ktanner@packrat.aml.arizona.edu

Jacques Evin

Centre de Datation par le RadioCarbone

Université Claude Bernard Lyon 1

43 Boulevard du 11 Novembre 1918

Bâtiment 217

F-69622 Villeurbanne CEDEX, France

Tel: 33724482 57; Fax: 3372431317

E-mail: jaques.evin@cismsun.univ-lyon1.fr

Yorgos Facorellis

Laboratory of Archaeometry

Institute of Materials Science

National Center for Scientific Research

"Demokritos"

15310 Aghia Paraskevi

P.O.B. 60288

Attiki, Greece

Tel: 30-1-65 13 111; Fax: 30-1-65 19430 
George W. Farwell

Nuclear Physics Laboratory GL-10

University of Washington

Seattle, Washington 98195 USA

Jiri Filip

Radiochemical Laboratory

The T.G. Masaryk Water Research Institute

Podbabska 30

CZ-160 62 Prague 6, Czech Republic

Tel: 422263 10757; Fax: 42224310450

Eberhard Finckh

Physikalisches Institut

Erwin-Rommel-Strasse 1

D-91054 Erlangen, Germany

Tel: 49-9131-857075; Fax: 49-9131-15249

John Flenley

Geography Department

Massey University

Palmerston North, New Zealand

Tel: 64-3569099; Fax: 64-3505644

E-mail: j.flenley@massey.ac.nz

Marc Fournier

Géochemie Isotopique

ORSTOM

72, Route d'Aulnay

F-93143 Bondy CEDEX, France

Tel: 33-1-48 02 56 36; Fax: 33-1-48 473088

E-mail: fournier@bondy.orstom.fr

Frank Fox

Canberra Packard Limited

Brook House

14 Station Road

Pangbourne

Berkshire, RG8 7DT, England

Jürgen C. Freundlich

Sudetenweg 5

D-50858 Köln, Germany

Tel: 49221686268

Klaus Froehlich

International Atomic Energy Agency

Wagramerstrasse 5

P.O. Box 100

A-1400 Vienna, Austria

Tel: 631 23601739; Fax: 631234564

E-mail: froehlik@ripo1.iaea.or.at
M. Fulker

Buttermore Pavilion

Westlakes Science \& Technology Park

Moor Row

Cumbria, CA24 3JZ, England

Tel: 44-1946 514000; Fax: 44-1965 514060

Menotti Galli

Dipartimento di Fisica

Università di Bologna

Via Irherio 46

I-40126 Bologna, Italy

José F. García-Martinez

Radiocarbon Laboratory

Departament de Química Analítica

Universitat de Barcelona

Avenida Diagonal, 647

E-08028 Barcelona, Spain

Tel: 34-3-402 12 81; Fax: 34-3-402 1233

Sharon Gedye

Department of Geography

University of Liverpool

Liverpool, L69 3BX, England

Tel: 44-151-744-2863; Fax: 44-151-744-2866

E-mail: chuckie@liverpool.ac.uk

Jorunn Gislefoss

Radiological Dating Laboratory

Norwegian Institute of Technology

N-7034 Trondheim, Norway

Tel: 47-73-593310; Fax: 47-73-593383

Vicente Gomez-Gil

CIEMAT

Ministerio de Industria y Energia

Avenida Complutense 22

E-28040 Madrid, Spain

Tel: 3466708; Fax: 3466121

Cecilio González-Gómez

Radioquimica

Departamento de Quimica Inorgánica

Facultad de Ciencias

Universidad de Granada

E-18071 Granada, Spain

Tel: 34582433 25; Fax: 3458243322

E-mail: cecilio@goliat.ugr.es 
Glenn Goodfriend

Geophysical Laboratory

Carnegie Institute of Washington

5251 Broad Branch Road, NW

Washington, DC 20015 USA

Tel: 202-686-2491; Fax: 202-686-2419

E-mail: goodfriend@ge.ciw.edu

Jochen Görsdorf

Deutsches Archäologisches Institut

Eurasien-Abteilung

${ }^{14} \mathrm{C}$ Labor

Leipziger Strasse 3/4

D-10117 Berlin, Germany

Tel/Fax: 49-30-20 377275

Tomasz Goslar

Radiocarbon Laboratory

Institute of Physics

Department of Radioisotopes

Silesian Technical University

Ul. Krzywoustego 2

PL-44-100 Gliwice, Poland

Tel/Fax: 4832372254

E-mail: goslar@zeus.polsl.gliwice.pl

Andreas Gottdang

High Voltage Engineering Europa B.V.

Amsterdamsweg 63

NL-3812 RR Amersfoort, The Netherlands

Tel: 31-33-619741; Fax: 31-33-615291

Anne Gravett

Department of Biology

Silwood Park

Ascot

Berkshire, England

Tel: 44-1344 294336; Fax: 44-1344 294339

Sheila Griffin

Radiocarbon Laboratory

Department of Earth System Science

University of California, Irvine

PSRF 207

Irvine, California 92717 USA

Tel: 714-824-3286; Fax: 714-824-3256

E-mail: sgriffin@uci.edu
Peter M. Grootes

Leibniz Labor für Altersbestimmung

und Isotopenforschung

Christian-Albrechts-Universität

Olshausentrasse 40-60

D-24118 Kiel, Germany

Tel: 49-431-880-3894; Fax: 49-431-880-3356

E-mail: pke26@rz.uni-kiel.d400.de

Rainer Grün

Quaternary Dating Research Centre

Australian National University

Research School of Pacific Studies

Canberra, ACT 0200 Australia

Tel: 61-6-249-3122; Fax: 61-6-249-0315

E-mail: rainer.grun@anu.edu.au

Steinar Gulliksen

Radiological Dating Laboratory

Norwegian Institute of Technology

N-7034 Trondheim, Norway

Tel: 47-73-593310; Fax: 47-73-593383

E-mail: sgull@phys.unit.no

Zhiyu Guo

Institute of Heavy Ion Physics

Peking University

Beijing 100871 China

Tel: 86-1-256 1166 x 5407;

Fax: 86-1-250 1875

E-mail: puihip@bepc2.ihep.ac.cn

Herbert Haas

Radiocarbon Laboratory

Water Resources Center

Desert Research Institute

P.O. Box 19040

Las Vegas, Nevada 89132-0040 USA

Tel: 702-895-0416; Fax: 702-895-0427

E-mail: haas@snsc.unr.edu

Laura Hainsworth

Center for Accelerator Mass Spectrometry (CAMS)

Lawrence Livermore National Laboratory

University of California

P.O. Box 808, L-397

Livermore, California 94550 USA

Tel: 510-423-1414; Fax:510-423-7884 
Irena Hajdas

Institut für Teilchenphysik

ETH-Hönggerberg

CH-8093 Zürich, Switzerland

Tel: 411633 2042; Fax: 4116331067

E-mail: hajdas@particle.phys.ethz.ch

Valerie A. Hall

Institute of Irish Studies

The Queen's University of Belfast

8 Fitzwilliam Street

Belfast, BT9 6AW, Northern Ireland

Tel: 44-1232 245133 x 3387;

Fax: 44-1232 439238

Douglas D. Harkness

NERC Radiocarbon Laboratory

Scottish Enterprise Technology Park

East Kilbride, G75 0QF, Scotland

Tel: 44-13522-60037; Fax: 44-13552-29829

E-mail: ek_dh@vaxa.nerc-murchison.ac.uk

Ron Hatfield

Beta Analytic, Inc.

4985 SW 74 Court

Miami, Florida 33155 USA

Tel: 305-667-5167; Fax: 305-663-0964

E-mail: beta@analytic.win.net

Jürg Hauenstein

Physikalisches Institut

Universität Bern

Sidlerstrasse 5

CH-3012 Bern, Switzerland

Tel: 4131631 4464; Fax: 41316314405

E-mail:merkt@phil.unibe.ch

John Head

Quaternary Dating Research Centre

Australian National University

Research School of Pacific Studies

Canberra, ACT 0200 Australia

Tel: 616249 0112; Fax: 6162490315

E-mail: mjh409@cscgpo.anu.edu.au

Magnus Hedberg

Swedish Museum of Natural History

Department of Mineralogy

Laboratory for Isotope Geology

Box 50007

S-104 05 Stockholm, Sweden

Tel: 468666 4067; Fax: 4686664031

E-mail: lig-magnus@nrm.se
Robert Hedges

Oxford Radiocarbon Accelerator Unit

Research Laboratory for Archaeology

and the History of Art

Oxford University

6 Keble Road

Oxford, OX1 3QJ, England

Tel: 44-1865 273939; Fax: 44-1865 273932

E-mail: orau@vax.ox.ac.uk

Susanne Heier-Nielsen

AMS ${ }^{14} \mathrm{C}$ Dating Laboratory

Institute of Physics and Astronomy

University of Aarhus

DK-8000 Aarhus C, Denmark

Tel: 45894237 36; Fax: 4586120740

E-mail: heier@dfi.aau.dk

Hendrik Heijnis

Quaternary Dating Research Centre

Australian National University

Research School of Pacific Studies

Canberra, ACT 0200 Australia

Tel: 61-6-249-3122; Fax: 61-6-249-0315

Jan Heinemeier

AMS ${ }^{14} \mathrm{C}$ Dating Laboratory

Institute of Physics and Astronomy

University of Aarhus

DK-8000 Aarhus C, Denmark

Tel: 458942 2899; Fax: 4586120740

E-mail:jh@dfi.aau.dk

Ede Hertelendi

Institute of Nuclear Research of the

Hungarian Academy of Sciences

P.O. Box 51

H-4001 Debrecen, Hungary

Tel: 3652 417266; Fax: 3652416181

E-mail: her@moon.atomki.hu

Vago Hesshaimer

Institut für Umweltphysik

Universität Heidelberg

Im Neuenheimer Feld 366

D-69120 Heidelberg, Germany

Tel: 496221 563357; Fax: 496221563405

E-mail:hr@uphys1.uphysn.uni-heidelberg.de 
Thomas Higham

Radiocarbon Dating Laboratory

University of Waikato

Private Bag 3105

Hamilton, New Zealand

Tel: 64-7-838 4278; Fax: 64-7-838 4192

E-mail: thigham@waikato.ac.nz or

http://www2.waikato.ac.nz/c14/

Achim Hiller

Arbeitsgruppe Paläoclimatologie

Quartärzentrum der Universität Leipzig

Permoserstrasse 15

D-04303 Leipzig, Germany

Tel: 49-341 325 2524; Fax: 49-341 2352576

Alan Hogg

Radiocarbon Laboratory

University of Waikato

Private Bag 3105

Hamilton, New Zealand

Tel: 64-7-838 4278; Fax: 64-7-838 4192

E-mail: ahogg@waikato.ac.nz

Stephen Hoper

Radiocarbon Dating Laboratory

Palaeoecology Centre

School of Geosciences

The Queen's University of Belfast

Belfast, BT7 1NN, Northern Ireland

Tel: 44-1232-335141; Fax: 44-1232-315779

Nada Horvatinčic

${ }^{14} \mathrm{C}$ and ${ }^{3} \mathrm{H}$ Laboratory

Rudjer Boß̌ković Institute

P.O.B. 1016, Bijenička 54

10001 Zagreb, Croatia

Tel: 38541 425-809; Fax: $38541425-497$

E-mail: horvatin@olimp.irb.hr

Mike Hotchkis

ANTARES AMS Centre

Australian Nuclear Science and Technology

New Illawarra Road

Lucas Heights, NSW 2234 Australia

Tel: 612 7173493; Fax: 6127179265

E-mail:mah@atom.ansto.gov.au

Rupert Housley

Department of Archaeology

University of Glasgow

Glasgow, G12 8QW, Scotland

Tel: 44-141 339 8855; Fax: 44-141 3078066
Richard Hyder

A.W. Wright Nuclear Structure Laboratory

Yale University

272 Whitney Avenue

New Haven, Connecticut 06511 USA

Tel: 203-432-3087; Fax: 203-432-3522

Marian Hyman

Department of Chemistry

Texas A \& M University

College Station, Texas 77843-3255 USA

Tel: 409-845-4084; Fax: 409-845-4729

Salvatore Improta

Dipartimento di Fisica

Università "Ld Sapienza"

Piazzale Aldo Moro, 2

I-00185 Rome, Italy

Tel: 39-6-4957697

Susan Ivy

Institut für Teilchenphysik

ETH-Hönggerberg

CH-8093 Zürich, Switzerland

Tel: 411633 2042; Fax: 4116331067

E-mail: ivy@eawag.ch

John Jirikowic

Radiocarbon Dating Laboratory

Palaeocology Centre

School of Geosciences

The Queen's University of Belfast

Belfast, BT7 1NN, Northern Ireland

Tel: 44-1232-335141 x 3128; Fax: 44-1232-321280

E-mail:jojirikowic@delphi.com

Glenn Jones

Texas Institute of Oceanography

Texas A \& M University

Galveston Branch

Galveston, Texas USA

Timothy Jull

NSF-Arizona AMS Facility

Department of Physics

The University of Arizona

Tucson, Arizona 85721 USA

Tel: 520-621-6816; Fax: 520-621-9619

E-mail: jull@u.arizona.edu 
Högne Jungner

Radiocarbon Dating Laboratory

Snellmaninkatu 3

P.O. Box 11

FIN-00014 Helsinki University, Finland

Tel: 358-0-1912-3436; Fax: 358-0-1912-3466

E-mail: hjungner@katk.helsinki.fi

Lauri Kaihola

Environmental Control Systems

Wallac Oy

Mustionkatu 6

P.O. Box 10

FIN-20101 Turku, Finland

Tel: 358-21-267 8376; Fax: 358-21-267 8357

E-mail: lkai@mail.wallac.fi

Tuovi Kankainen

Geological Survey of Finland

FIN-02150 Espoo, Finland

Tel: 358-0-46931; Fax: 358-0-462205

E-mail: tuovi.kankainen@gsf.fi

Michaele Kashgarian

Center for Accelerator Mass Spectrometry (CAMS)

Lawrence Livermore National Laboratory

University of California

P.O. Box 808, L-397

Livermore, California 94550 USA

Tel: 510-423-1414; Fax: 510-423-7884

Michael Kessler

Packard Instrument Company

800 Research Parkway

Meriden, Connecticut 06450 USA

Tel: 203-238-2351; Fax: 203-639-2172

Kunihiko Kigoshi

Radiocarbon Laboratory

Gakushuin University

Mejiro

Toshimaku-ku, 1-5-1, Faculty of Science

Tokyo 171 Japan

Tel: 81-3-986-0221 x 482

Fax: 81-3-5992-10029

E-mail: hori@se.shibaura-it.ac.jp

Mark Kilian

Center for Isotope Research

University of Groningen

Nijenborgh 4

NL-9747 AG, Groningen, The Netherlands

Tel: 31-50-634760; Fax: 31-50-634738

E-mail: kilian@sara.nl
Donna Kirner

Radiocarbon Laboratory

Department of Anthropology

University of California, Riverside

Riverside, California 92521-1418 USA

Tel: 909-787-5521; Fax: 909-787-5409

Hiroyuki Kitagawa

International Research Center for Japanese Studies

3-2 Oeyama-cho

Goryo, Nishikyo-ku

Kyoto 610-11 Japan

Tel: 81-75-335-2100; Fax: 81-75-335-2090

E-mail: kitaga@nichibun.ac.jp

George Klouda

National Institute of Standards \& Technology

Room B364, Building 222

Gaithersburg, Maryland 20899 USA

Tel: 301-975-3919; Fax: 301-975-3845

Atte Korhola

Department of Geography

Laboratory of Physical Geography

P.O. Box 9

FIN-00014 University of Helsinki, Finland

Tel: 358-0-1911; Fax: 358-0-191 8670

E-mail: korhola@penger.helsinki.fi

Nikolaj Kovalyukh

Institute of Environmental Radiogeochemistry

Ukrainian Academy of Sciences

Paladina 34

Kiev 252142 Ukraine

Tel/Fax: 7-044-444-0060 or 7-044-444-0105

Renee Kra

RADIOCARBON

Department of Geosciences

The University of Arizona

4717 E. Ft. Lowell Road

Tucson, Arizona 85712 USA

Tel: 520-881-0857; Fax: 520-881-0554

E-mail: rkra@packrat.aml.arizona.edu

Bernd Kromer

Heidelberger Akademie der Wissenchaften

c/o Institut für Umweltphysik

Im Neuenheimer Feld 366

D-69120 Heidelberg, Germany

Tel: 49-6221-563359; Fax: 49-6221-563405

kr@uphys1.uphysn.uni-heidelberg.de 
Tadeusz Kuc

Kraków Radiocarbon Laboratory

Environmental Physics Department

The Academy of Mining and Metallurgy

Al. Mickiebicza 30

PL-30-059 Kraków, Poland

Tel: 48-12-333740; Fax: 48-12-334998

E-mail: kuc@vsko1.ifj.edu.pl

Sheela Kusumgar

Radiocarbon Dating Research Unit

Physical Research Laboratory

Navrangpura

Ahmedabad 380009 India

Tel: 91-272-462129; Fax: 91-272-460502

Todd Lange

NSF-Arizona AMS Facility

Department of Physics

The University of Arizona

Tucson, Arizona 85721 USA

Tel: 520-621-6810; Fax: 520-621-9619

E-mail: lange@soliton.physics.arizona.edu

Jan Lanting

Biologisch-Archeologisch Instituut

Poststraat 6

NL-9712 ER, Groningen, The Netherlands

Tel: 50 636712; Fax: 50636992

Oliver Lavery

Radiocarbon Dating Laboratory

Palaeocology Centre

School of Geosciences

The Queen's University of Belfast

Belfast, BT7 1NN, Northern Ireland

Tel: 44-1232-335141; Fax: 44-1232-315779

Ewan Lawson

ANTARES AMS Centre

Australian Nuclear Science and Technology

New Illawarra Road

Lucas Heights, NSW 2234 Australia

Tel: 612 7173493; Fax: 6127179265

E-mail: eml@nucleus.ansto.gov.au

Steven W. Leavitt

Laboratory of Tree-Ring Research

The University of Arizona

Tucson, Arizona 85721 USA

Tel: 520-621-6468; Fax: 520-621-8229

E-mail: sleavitt@ccit.arizona.edu
Jin Li

${ }^{14} \mathrm{C}$ Laboratory

Institute of Geography

Chinese Academy of Sciences

Beijing 100101 China

Tel: 861 6917242; Fax: 8616911844

Jiun-Chuan Lin

Department of Geography

National Taiwan University

Taipei 106 Taiwan, Republic of China

Tel: 886-2-368 7056; Fax: 886-2-368-7056

E-mail: jclin@ccms.ntu.edu.tw

\section{Cliff Litton}

Department of Mathematics

University of Nottingham

University Park

Nottingham, NG7 2RD, England

Tsung-Kwei Liu

Department of Geology

National Taiwan University

245 Choushan Road

Taipei 10770 Taiwan, Republic of China

Tel/Fax: 886-2-365 7380

E-mail: liutk@sun03.gl.ntu.edu.tw

Gro Locka

Radiological Dating Laboratory

Norwegian Institute of Technology

N-7034 Trondheim, Norway

Tel: 47-73-593310; Fax: 47-73-593383

Austin Long

Laboratory of Isotope Geochemistry

Department of Geosciences

The University of Arizona

Tucson, Arizona 85721 USA

Tel: 520-621-8888; Fax: 520-621-2672

E-mail: along@geo.arizona.edu

Heinz Hugo Loosli

Physikalisches Institut

Universität Bern

Sidlerstrasse 5

CH-3012 Bern, Switzerland

Tel: 4131631 4464; Fax: 41316314405

E-mail: merkt@phil.unibe.ch 
John Lowe

Centre for Quaternary Research

Geography Department

Royal Holloway

University of London

Egham, Surrey, TW20 0EX, England

Tel: 44-1784 443565; Fax: 44-1784 672836

Bernard Maloney

Radiocarbon Dating Laboratory

Palaeocology Centre

The Queen's University of Belfast

Belfast, BT7 1NN, Northern Ireland

Tel: 44-1232-245133 x 5143; Fax: 44-1232-321280

E-mail: b.k.maloney@queens-belfast.ac.uk

Yannis Maniatis

Laboratory of Archaeometry

NCSR Demokritos

15310 Aghia Paraskevi

Attiki, Greece

Toshiyuki Masuzawa

Institute for Hydrospheric-Atmospheric Sciences

Nagoya University

Chikusa-ku

Nagoya 464-01 Japan

Tel: 8152789 3698; Fax: 81527893449

E-mail: g44525a@nucc.cc.nagoya-u.ac.jp

Martin McCartney

Radiocarbon Dating Laboratory

Scottish Universities Research \& Reactor Centre

(SURRC)

Scottish Enterprise Technology Park

East Kilbride, G75 0QF, Scotland

Tel: 44-13552-70163; Fax: 44-13552-29898

Gerry McCormac

Radiocarbon Dating Laboratory

Palaeoecology Centre

School of Geosciences

The Queen's University of Belfast

Belfast, BT7 1NN, Northern Ireland

Tel: 44-1232-335141; Fax: 44-1232-315779

E-mail: pag0409@queens-belfast.ac uk or

f.mccormac@queens-belfast.ac.uk

Jim McDonald

Radiocarbon Dating Laboratory

Palaeoecology Centre

School of Geosciences

The Queen's University of Belfast

Belfast, BT7 1NN, Northern Ireland

Tel: 44-1232-335141; Fax: 44-1232-315779
P. McDonald

Buttermore Pavilion

Westlakes Science and Technology Park

Moor Row

Cumbria, CA24 3JZ, England

Tel: 44-1946 516000; Fax: 44-1966 514060

Lanny McHargue

Department of Geosciences

The University of Arizona

Tucson, Arizona 85721 USA

Tel: 520-621-6024; Fax: 520-621-2672

E-mail: mchargue@physics.arizona.edu

Joseph McKee

Rafter Radiocarbon Laboratory

Institute of Geological and Nuclear Sciences

P.O. Box 31312

Lower Hutt, New Zealand

Tel: 64-4-570 4671; Fax: 64-4-569 0657

E-mail: j.mckee@gns.cri.nz

Ann McNichol

National Ocean Sciences AMS Facility

Woods Hole Oceanographic Institution

Woods Hole, Massachusetts 02543 USA

Tel: 508-289-3394 or 508-457-2000 x 3394

Fax: 508-457-2183

E-mail: amcnichol@whoi.edu

Harro Meijer

Center for Isotope Research

University of Groningen

Nijenborgh 4

NL-9747 AG, Groningen, The Netherlands

Tel: 31-50 634760; Fax: 31-50 634738

E-mail: meijer@phys.rug.nl

Niklas Meinander

Radiocarbon Dating Laboratory

Snellmaninkatu 3, P.O. Box 11

FIN-00014 Helsinki University, Finland

Tel: 358-0-1912-3436; Fax: 358-0-1912-3466

E-mail: meinander@phcu.helsinki.fi

Joan S. Mestres

Radiocarbon Laboratory

Departament de Química Analítica

Universitat de Barcelona

Avenida Diagonal, 647

E-08028 Barcelona, Spain

Tel: 34-3 402 12 81; Fax: 34-3 4021233 
Adam Michczyński

Radiocarbon Laboratory

Institute of Physics

Department of Radioisotopes

Silesian Technical University

Ul. Krzywoustego 2

PL-44-100 Gliwice, Poland

Tel/Fax: 4832372254

michczynski@gleto2.gliwice.edu.pl

Alice Mignerey

Department of Chemistry

University of Maryland

College Park, Maryland 20742 USA

Tel: 301-605-1852; Fax: 301-314-9121

E-mail: 201228@umdd.umd.edu

Brian Miller

NERC Radiocarbon Laboratory

Scottish Enterprise Technology Park

East Kilbride, G75 0QF, Scotland

Tel: 44-13522-60037; Fax: 44-13552-29829

Gwen Milton

AECL Research

Health and Environmental Sciences Division

Chalk River Laboratories

Chalk River, Ontario, K0J 1J0, Canada

Tel: 613-584-3311; Fax: 613-584-4024

E-mail: miltong@erl.aecl.ca

Willem Mook

Netherlands Institute for Sea Research (NIOZ)

P.O. Box 59

NL-1790 AB Den Burg

Texel, The Netherlands

Tel: 31-2220-69300; Fax: 31-2220-19674

E-mail: texel@nioz.nl

Janine Moore

Radiocarbon Dating Laboratory

Palaeoecology Centre

School of Geosciences

The Queen's University of Belfast

Belfast BT7 1NN Northern Ireland

Tel: 44-1232-335141; Fax: 44-1232-315779

Toshio Nakamura

Dating and Materials Research Center

Nagoya University

Chikusa

Nagoya 464-01 Japan

Tel: 81-52-789-2578; Fax: 81-52-789-3095

E-mail: g44466a@nucc.cc.nagoya-u.ac.jp
Philip Naysmith

Radiocarbon Dating Laboratory

Scottish Universities Research \& Reactor Centre

(SURRC)

Scottish Enterprise Technology Park

East Kilbride, G75 0QF, Scotland

Tel: 44-13552-23332; Fax: 44-13552-29898

Erle Nelson

Archaeology Department

Simon Fraser University

Burnaby, British Columbia, V5A 1S6, Canada

Tel: 604-291-3673; Fax: 604-291-5666

E-mail: erle-nelson@sfu.ca

Thomas R. Niklaus

Institute für Teilchenphysik

ETH-Hönggerberg

CH-8093 Zürich, Switzerland

Tel: 41163365 05; Fax: 4116331067

E-mail: niklaus@imp.phys.ethz.ch

John E. Noakes

Center for Applied Isotope Studies (CAIS)

The University of Georgia

120 Riverbend Road

Athens, Georgia 30602-4702 USA

Tel: 706-542-1395; Fax: 706-542-6106

Reidar Nydal

Radiological Dating Laboratory

Norwegian Institute of Technology

N-7034 Trondheim, Norway

Tel: 47-73-593310; Fax: 47-73-593383

E-mail: nydalr@phys.unit.no

R. O'Donnell

Experimental Physics Department

University College Dublin

Bellfield

Dublin, Eire

Tel: 3531706 2220; Fax: 35312837275

Patrick E. O'Sullivan

Department of Environmental Sciences

University of Plymouth

Plymouth, PL4 8AA, England

E-mail: p05118@plym-pa.ac.uk 
Bogomil Obelić

${ }^{14} \mathrm{C}$ and ${ }^{3} \mathrm{H}$ Laboratory

Rudjer Bošković Institute

P.O. Box 1016, Bijenicka 54

10000 Zagreb, Croatia

Tel: 385-1-425-809; Fax: 385-1-425-497

E-mail: obelic@olimp.irb.hr

Christine Oberlin

Centre de Datation par le RadioCarbone

Université Claude Bernard Lyon 1

43 Boulevard du 11 Novembre 1918

Bâtiment 217

F-69622 Villeurbanne CEDEX, France

Tel: 337244 8257; Fax: 3372431317

\section{Neil Ogle}

Radiocarbon Dating Laboratory

Palaeoecology Centre

School of Geosciences

The Queen's University of Belfast

Belfast, BT7 1NN, Northern Ireland

Tel: 44-1232-335141; Fax: 44-1232-315779

Ingrid Olsson

Department of Physics

Uppsala University

Box 530

S-751 21 Uppsala, Sweden

Tel: 46181835 71; Fax: 4618183524

E-mail: ingrid.olsson@fysik.uu.se

Kunio Omoto

Radiocarbon Dating Laboratory

Department of Geography

College of Humanities and Science

Nihon University

25-40, 3 Chome, Sakurajosui

Setagaya-Ku, Tokyo 156 Japan

Tel: 81-35317-9723 or 81-33303-1691

Fax: 81-33303-9899

Robert L. Otlet

Radiocarbon Dating

The Old Stables

East Lockinge

Wantage, Oxon, OX12 8QY, England

Tel/Fax: 44-1235 833667

Barbara Ottaway

Department of Prehistory and Archaeology

University of Sheffield

Sheffield, S10 2TN, England

Tel: 44-1762 826082; Fax: 44-1762 722563
Edwin Pak

Institut für Radiumforschung und Kernphysik

Universität Wein

Boltzmanngasse 3

A-1090 Vienna, Austria

Tel: 43131367 3514; Fax: 431313673502

Anna Pazdur

Radiocarbon Laboratory

Institute of Physics

Department of Radioisotopes

Silesian Technical University

Ul. Krzywoustego 2

PL-44-100 Gliwice, Poland

Tel/Fax: 4832372254

E-mail: anna@fizyka.polsl.gliwice.pl

M. F. Pazdur ${ }^{\dagger}$

Radiocarbon Laboratory

Institute of Physics

Department of Radioisotopes

Silesian Technical University

Ul. Krzywoustego 2

PL-44-100 Gliwice, Poland

Tel/Fax: 4832372254

J. Douglas Peacock

18 McLaren Road

Edinburgh, EH9 2BN, Scotland

Tsung-Hung Peng

Ocean Chemistry Division

NOAA/AOML/OCD

4301 Rickenbacker Causeway

Miami, Florida 33149 USA

Tel: 305-361-4399; Fax: 305-361-4582

E-mail:peng@aoml.erl.gov

Daniel Penny

Department of Geography and

Environmental Sciences

Monash University

Victoria, Australia

Tel: 63905 2919; Fax: 639052968

Luiz C. R. Pessenda

Center for Nuclear Energy in Agriculture (CENA)

University of São Paulo

Avenida Centenario 303

P.O. Box 96-13418-260

Piracicaba SP, Brazil

Tel: 55-194-33-5122; Fax: 55-194-22-8339

E-mail: lcrpesse@pira.cena.usp.br

†Deceased 11 May 1995 
Jon R. Pilcher

Radiocarbon Dating Laboratory

Palaeoecology Centre

School of Geosciences

The Queen's University of Belfast

Belfast, BT7 1NN, Northern Ireland

Tel: 44-1232 2451335; Fax: 44-1232 321280

E-mail: j.pilcher@queens-belfast.ac.uk

Henry A. Polach

Research Consultations

P.O. Box 43

Garran ACT 2605 Australia

Tel: 616281 2592; Fax: 6162812592

E-mail: henry.polach@anu.edu.au

Göran Possnert

The Svedberg Laboratory

University of Uppsala

Box 533

S-751 21 Uppsala, Sweden

Tel: 4618 183059; Fax: 4618155736

Pavel P. Povinec

International Atomic Energy Agency

Marine Environment Laboratory

19 av. de Castellans

B.P. 800

MC-98012 Monaco CEDEX

Tel. 33920522 22; Fax 3392057744

E-mail: povinec@unice.fr

Christine Prior

Radiocarbon Laboratory

Department of Anthropology

University of California, Riverside

Riverside, California 92521 USA

Tel: 909-787-5521; Fax: 909-787-5409

E-mail: cprior@ucrac1.ucr.edu

Jaan-Mati Punning

Institute of Ecology

Paldiski 1

EE-0101 Tallinn, Estonia

Tel: 3722451 634; Fax: 3722453748

Kenneth Purser

Southern Cross Corporation

30A Cherry Hill Drive

Danvers, Massachusetts 01923 USA

Tel: 508-750-8455; Fax: 508-774-0940

E-mail: kenpurser@eworld.com
Uffe Rahbek

C-14 Dating Laboratory

Nr. Vestergade 11

DK-1471 Copenhagen, Denmark

E-mail: c14-ur@palais.natmus.min.dlc

Grant M. Raisbeck

CSNSM

Bâtiment 108

F-91405 Campus Orsay, France

Tel: 33-1-6941-5264; Fax: 33-1-6941-5268

E-mail: lestring@frcpnll.in2p3.fr

Raivo Rajamae

Institute of Geology

Estonian Academy of Sciences

7 Estonia Avenue

EE-0100 Tallinn, Estonia

Tel: 3722538 378; Fax: 3726312074

E-mail: rajamae@isogeo.gi.ee

Christopher Bronk Ramsey

Oxford Radiocarbon Accelerator Unit

Research Laboratory for Archaeology and the History of Art

University of Oxford

6 Keble Road

Oxford, OX1 3QJ, England

Tel: 44-1865 273939; Fax: 44-1865 273932

E-mail: orau@vax.ox.ac.uk

Paula J. Reimer

Quaternary Research Center AK-60

Department of Geological Sciences

University of Washington

Seattle, Washington 98195 USA

Tel: 206-543-6327; Fax: 206-543-3191

E-mail: pjreimer@u.washington.edu

R. Reuter

c/o Prof. A. Mignerey

Department of Chemistry

University of Maryland

College Park, Maryland 20740 USA

Frank Rickey

Department of Physics

Building 1396

Purdue University

West Lafayette, Indiana 47907 USA

Tel: 317-494-5535; Fax: 317-494-0706

E-mail: far@primelab.physics.purdue.edu 
Steve Robertson

Quaternary Dating Research Centre

Research School of Pacific Studies

Australian National University

Canberra, ACT 0200 Australia

Tel: 616249 4035; Fax: 6162490315

E-mail: steve.robertson@anu.edu.au

Stephen W. Robinson

237 Marmona Drive

Menlo Park, California 94025 USA

Tel: 415-328-2077; Fax: 415-325-2077-3*

Marvin W. Rowe

Department of Chemistry

Texas A \& M University

College Station, Texas 77843-3255 USA

Tel: 409-845-1929; Fax: 409-845-4729

E-mail: rowe@chemvx.tamu.edu

Meyer Rubin

U.S. Geological Survey

National Center 971

Reston, Virginia 22092 USA

Tel: 703-648-5350; Fax: 703-648-5310

Niels Rud

AMS ${ }^{14} \mathrm{C}$ Dating Laboratory

Institute of Physics and Astronomy

University of Aarhus

DK-8000 Aarhus C, Denmark

Tel: 458942 2899; Fax: 4586120740

David Sanderson

Radiocarbon Dating Laboratory

Scottish Universities Research \& Reactor Centre (SURRC)

Scottish Enterprise Technology Park

East Kilbride, G75 0QF, Scotland

Tel: 44-13552-23332; Fax: 44-13552-29898

Robert J. Schneider

National Ocean Sciences AMS Facility

McLean Laboratory

Woods Hole Oceanographic Institution

Woods Hole, Massachusetts 02543 USA

Tel: 508-457-2000 x 2585; Fax: 508-457-2183

E-mail: rjs@ams265.whoi.edu
Marian Scott

Department of Statistics

University of Glasgow

University Gardens

Glasgow, G12 8QW, Scotland

Tel: 44-141-339-8855; Fax: 44-141-330-4814

E-mail:marian@stats.gla.ac.uk

Dror Segal

Department of Environmental Science and

Energy Research

Weizmann Institute of Science

76100 Rehovot, Israel

Tel: 9728 342544; Fax: 9728344124

Mike Shepherd

Department of Geography

Massey University

Private Bag 11222

Palmerston North, New Zealand

Tel: 64-6356 9099 x 7465; Fax: 64-6350 5644

E-mail:m.shepherd@massey.ac.nz

Jennifer Shore

Radiocarbon Dating Laboratory

Scottish Universities Research \& Reactor

Centre (SURRC)

Scottish Enterprise Technology Park

East Kilbride, G75 0QF, Scotland

Tel: 44-13552-23332; Fax: 44-13552-29898

Jan Silar

Institute of Hydrogeology, Engineering

Geology and Applied Geophysics

Faculty of Science

Albertov 6

Charles University

12843 Praha 2, Czech Republic

Tel: 42-2-24 9154 72; Fax: 42-2-29 6084

Goran Skog

Radiocarbon Dating Laboratory

University of Lund

Tornavägen 13

S-223 63 Lund, Sweden

Tel: 46-46-107885; Fax: 46-46-104830

E-mail: goran.skog@geol.lu.se

Vadim Skripkin

Institute of Environmental Radiogeochemistry

Ukrainian Academy of Sciences

Paladina 34

Kiev 252142 Ukraine

Tel/Fax: 7-044-444-0060 or 7-044-444-0105 
Andrew Smith

ANTARES AMS Centre

Australian Nuclear Science and Technology

New Illawarra Road

Lucas Heights, NSW 2234 Australia

Tel: 2717 9054; Fax: 27179265

E-mail: ams@photon.ansto.gov.au

A. M. Monge Soares

Laboratório de Isótopos Ambientas

Departamento de Química

ICEN-INETI

P-2685 Sacavém, Portugal

Harry Sobel $^{\dagger}$

Isotope and Archeometry Laboratory

Institute of Geophysics \& Planetary Physics

University of California, Los Angeles

Los Angeles, California 90024 USA

Eloni Sonninen

Radiocarbon Dating Laboratory

Snellmaninkatu 3

P.O. Box 11

FIN-00014 Helsinki University, Finland

Tel: 358-0-1912-3436; Fax: 358-0-1912-3466

E-mail: esonninen@cc.helsinki.fi

John Southon

Center for Accelerator Mass

Spectrometry (CAMS)

Lawrence Livermore National Laboratory

University of California

Box 808, L-397

Livermore, California 94550 USA

Tel: 510-423-4226; Fax: 510-423-7884

E-mail: southon@pagoda.llnl.gov

Petrit Spahiu

Radiochemistry and Tracers Division

Institute of Nuclear Physics

Tirana, Albania

Rodger Sparks

Rafter Radiocarbon Laboratory

Nuclear Sciences Group

Institute of Geological and Nuclear Sciences, Ltd.

P.O. Box 31-312

Lower Hutt, New Zealand

Tel: 64-4-570 4671; Fax: 64-4-569 0657

E-mail: r.sparks@gns.cri.nz
J.D. Spaulding

Center for Applied Isotope Studies (CAIS)

The University of Georgia

120 Riverbend Road

Athens, Georgia 30602-4702 USA

Tel: 706-542-1395; Fax: 706-542-6106

Harald Stauble

Landesamt für Archäologie-Arbeitsstelle

Braunkohle

Leipzegerstrasse 148

D-04442 Zwenkau, Germany

Tel/Fax: 49-34203-32569

Michael Stenhouse

INTERA

Parkview House

14B Burton Street

Melton Mowbray, England

Tel: 44-1664 411 445; Fax: 44-1664 411402

Minze Stuiver

Quaternary Isotope Laboratory AJ-20

Department of Geological Sciences

University of Washington

Seattle, Washington 98195 USA

Tel: 206-685-1735; Fax: 206-543-3836

E-mail: minze@u.washington.edu

Axel Suckow

Niedersächsisches Landesamt für

Bodenforschung

Abt. 1-Postfach 510153

Stilleweg 2

D-30655 Hannover 51 Germany

Tel: 49-511 643 2537; Fax: 49-511 6432304

E-mail: suckow@gate1.bgr.d400.de

Ámý E. Sveinbjörnsdóttir

Science Institute

University of Iceland

Dunhaga 3

IS-107 Reykjavík, Iceland

Tel: 354-1-694800; Fax: 354-1-28911

Ivo Svetlik

Radiochemical Laboratory

The T.G. Masaryk Water Research Institute

Podbabska 30

CZ-160 62 Prague 6, Czech Republic

Tel: 422263 10757; Fax: 42224310450

†Deceased 25 August 1994 
V.R. Switsur

Godwin Laboratory

University of Cambridge

Free School Lane

Cambridge, CB2 3RS, England

Tel: 44-1223-334877; Fax: 44-1223-334748

E-mail: vrs1@phx.cam.ac.uk

Carla Taricco

Istituto di Cosmogeofisica del CNR

Corso Fiume 4

I-10133-Torino, Italy

Tel: 39-11 660 4064; Fax: 39-11 6604056

Gillian Taylor

NWG MacIntosh Centre for Quaternary Dating

Madsen Building F09

University of Sydney

Sydney, NSW 2006 Australia

Tel: 61-2-692 3993; Fax: 61-2-552 1967

Claude B. Taylor

Rafter Radiocarbon Laboratory

Nuclear Sciences Group

Institute of Geological and Nuclear Sciences, Ltd.

P.O. Box 31-312

Lower Hutt, New Zealand

Tel: 64-4-570 4671; Fax: 64-4-5690657

E-mail: taylor@upo.gns.cri.nz

R.E. Taylor

Department of Anthropology

Institute of Geophysics and Planetary Physics

University of California, Riverside

Riverside, California 92512 USA

Tel: 909-787-5521; Fax: 909-787-5409

E-mail: retaylor@ucrvms

Roland Tesch

BFPZ Arsenal

Geotechnisches Institut

Abt. Isotopengeophysik

Postfach 8

A-1031 Vienna, Austria

Tel: 431797 67516; Fax: 43179767592

Pall Theodorsson

Science Institute

University of Iceland

Dunhaga 3

IS-107 Reykjavík, Iceland

Tel: 354-5694800; Fax: 354-5528911

E-mail: pth@raunvis.hi.is
John Thomson

Southampton Oceanography Centre

Empress Dock

Southampton, SO14 3ZH, England

Tel: 44-1703 596 548; Fax: 44-1703 596554

Nadine Tisnerat

Centre des Faibles Radioactivités

Avenue de la Terrasse

F-91198 Gif sur Yvette CEDEX, France

Tel: 331698235 25; Fax: 33169823568

Rolf Trettin

UFZ Leipzig-Halle

Sektion Hydrogeologie

Hallesche Strasse 44

D-06246 Bad Lauchstadt, Germany

Tel: 49-366 352 0355; Fax: 49-366 3520229

Scott Tucker

CARE

Silwood Park

Buckhurst Road

Ascot

Berkshire, SL5 7TE, England

Tel: 44-1364 294305; Fax: 44-1244 29431

Claudio Tuniz

ANTARES AMS Centre

Australian Nuclear Science and Technology

New Illawarra Road

Lucas Heights, NSW 2234 Australia

Tel: 612 7173493; Fax: 6127179265

tuniz@atom.ansto.gov.au

Gyorgy Uchrin

Institute of Isotopes of the Hungarian

Academy of Sciences

P.O. Box 77

H-1525 Budapest, Hungary

Tel: 361155 2694; Fax: 3611565045

Klaas van der Borg

van de Graaff Laboratorium

Rijksuniversiteit Utrecht

Princetonplein 5

P.O. Box 80.000

NL-3508 TA Utrecht, The Netherlands

Tel: 31-30-53 238; Fax: 31-30-51 689

E-mail: k.vanderborg@fus.ruu.nl 
L. van der Plaetsen

Archeolabs

Le Chatelard

F-38840 St. Bonnet de Chavagne, France Tel: 33-76 6400 97; Fax: 33-76 640160

Hans van der Plicht

Center for Isotope Research

Nijenborgh 4

NL-9747 AG, Groningen, The Netherlands

Tel: 31-50-634760; Fax: 31-50-634738

E-mail: plicht@phys.rug.nl

Gert J. van Klinken

Oxford Radiocarbon Accelerator Unit

Research Laboratory for Archaeology

and the History of Art

Oxford University

6 Keble Road

Oxford, OX1 3QJ, England

E-mail: orau@vax.ox.ac.uk

Henri van Oosterhout

High Voltage Engineering Europa

P.O. Box 99

NL-3800 AB Amersfoort, The Netherlands

Job J. van Roijen

Department of Subatomic Physics

Rijksuniversiteit Utrecht

P.O. Box 80.000

NL-3508 TA Utrecht, The Netherlands

Tel: 31-30-531656; Fax: 31-30-518689

E-mail: roijen@fys.ruu.nl

Mark Van Strydonck

Royal Institute of Cultural Heritage

Jubelpark 1

B-1040 Brussels, Belgium

Tel: 32-2-739 67 11; Fax: 32-2-732 0105

Mihaly Veres

Institute of Nuclear Research of the Hungarian

Academy of Sciences

P.O. Box 51

H-4001 Debrecen, Hungary

Tel: 3652417 266; Fax: 3652416181

E-mail: 49602ver@huella.bitnet

Michael Verkouteren

National Institute of Standards \& Technology

Room B364, Building 222

Gaithersburg, Maryland 20899 USA

Tel: 301-975-3919; Fax: 301-975-3845

E-mail: verkout_m@enh.nist.gov
John C. Vogel

Quaternary Dating Research Unit (QUADRU)

EMATEK-CSIR

P.O. Box 395

Pretoria 0001, South Africa

Tel: 2712841 3380; Fax: 27128041354

E-mail: jvogel@csir.co.za

John S. Vogel

Center for Accelerator Mass

Spectrometry (CAMS)

Lawrence Livermore National Laboratory

University of California

Box 808, L-397

Livermore, California 94550 USA

Tel: 510-423-4232; Fax: 510-423-7884

E-mail: john.vogel@quickmail.llnl.gov

Karl von Reden

National Ocean Sciences AMS Facility

McLean Laboratory

Woods Hole Oceanographic Institution

Woods Hole, Massachusetts 02543 USA

Tel: 508-457-2000 x 2585; Fax: 508-457-2183

E-mail: kvr@ams245.whoi.edu

A. J. Walker

Radiocarbon Dating

The Old Stables

East Lockinge

Wantage, Oxon OX12 8QY, England

Tel/Fax: 44-1235833667

Bernhard Weninger

Radiocarbon Laboratory

Institut der Ur-und Frühgeschichte

Universität zu Köln

Weyertal 125

D-50923 Köln, Germany

Tel: 49-221-470-2880; Fax: 49-221-470-4892

Alex Wilson

Department of Geosciences

The University of Arizona

Tucson, Arizona 85721 USA

Tel: 520-621-2986; Fax: 520-621-2672

Jenny Wilson

Radiocarbon Dating Laboratory

Palaeoecology Centre

School of Geosciences

The Queen's University of Belfast

Belfast, BT7 1NN, Northern Ireland

Tel: 44-1232-335141; Fax: 44-1232-315779

E-mail: pag0003@queens-belfast.vax2 
Ann Wintle

Luminescence Laboratory

Institute of Earth Studies

University of Wales, Aberystwyth

Penglais Campus

Aberystwyth

Dyfed, SY23 3DB, Wales

Barbara Wohlfarth

Department of Quaternary Geology Lunds Universitet

Tornavägen 13

S-223 63 Lund, Sweden

Tel: 46-107000; Fax: 46-46-104830

E-mail: barbara@gemini.ldc.lu.se

Francoise Yiou

Bâtiment 108, CSNSM

F-91405 Campus Orsay, France

Tel: 33-1-6941-5264; Fax: 33-1-6941-5268

E-mail: lestring@frcpnll.in2p3.fv

\section{Ganna Zaitseva}

Institute of the History of Material Culture

Dvortsovaya nab. 18

St. Petersburg 191065, Russia

Tel: 7-812-311-81-56; Fax: 7-812-311-62-71

E-mail:man@manuser.peter.ru

Albert Zondervan

Rafter Radiocarbon Laboratory

Nuclear Sciences Group

Institute of Geological and Nuclear Sciences, Ltd.

P.O. Box 31-312

Lower Hutt, New Zealand

Tel: 64-4-570 4671; Fax: 64-4-5690657 